\title{
Design and Implementation of Underwater Cable Inductive Coupling Communication System
}

\author{
Wei Xue ${ }^{a}$, Qingdong Ding ${ }^{b}$, Yujia Liu ${ }^{\mathrm{c}}$ \\ School of Information and Communication Engineering, Harbin Engineering University, Harbin \\ 150001, China \\ axuewei@hrbeu.edu.cn, bdingqingdong@ hrbeu.edu.cn, cliuyujia@ hrbeu.edu.cn
}

Keywords: Inductive Coupling, FPGA, Communication System.

\begin{abstract}
With the development of human society, the importance of the ocean is gradually being excavated. In order to transfer the data of underwater observation instrument to the water surface, the method of inductive coupling data transmission mode can be used. In this paper, the basic principle of the system was derived. This system used FPGA as the core to achieve the modulation and demodulation, and achieved the demand for inductive coupling communication with external circuit, it could have a certain practical value.
\end{abstract}

\section{Introduction}

Traditionally, underwater-to-surface data transmission was accomplished using direct cable connections. Such cables are bulky, expensive, and unreliable, and the positions of individual sensors are fixed once the cable is designed and manufactured [1,2]. Underwater wireless communication can be realized by acoustic remote sensing technology, but the channel environment is complex, which makes the system more complex and expensive. Underwater cable inductive coupling communication technology is a convenient, economic, reliable and flexible solution [3], its information transmission rate is higher than the underwater acoustic communication, and it has better real-time performance. Besides, the position of terminal equipment could be changed without a wired connection with cable [4]. Compared with the acoustic data transmission mode or cable communication mode, the performance of inductive coupling communication system is superior, the price is moderate, and the structure can be changed, it has been more and more widely used in recent years. In this paper, based on the principle of electromagnetic induction, the feasibility of inductive coupling communication was deduced, and the experimental verification was carried out.

\section{Principle of Inductive Coupling Communication}

The structure of inductive coupling communication is shown in Figure 1, and its equivalent circuit diagram is shown in Figure 2.

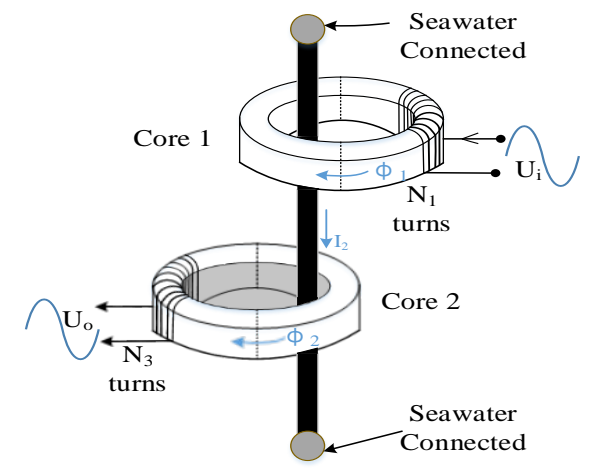

Fig. 1 Structure of inductive coupling

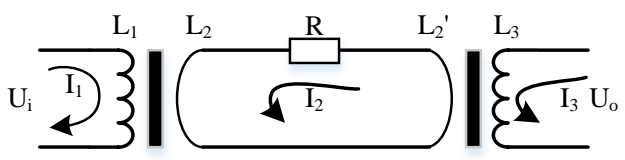

Fig. 2 Equivalent circuit diagram

The primary winding of magnetic ring 1 is excited by a carrier signal $U_{i}$ that contains the measured data, and a current is induced in the single-turn circuit, the cable is connected in series 
with the sea water as shown in Figure 1. The current in turn induces an electromotive force $U_{o}$ on the secondary winding of the magnetic ring 2, it obtains the data transmitted underwater through the demodulation of the water terminal. The main flux $\Phi_{1}$ in the magnetic ring is composed of the magnetic flux generated by the input winding and the single cable passing through the magnetic loop. Assuming that the induced electromotive force on the inductive coupling cable is $\mathrm{E}_{2}$, the loop composed of cable and sea water is shown in Figure 3, where $U_{2}=E_{2}$.

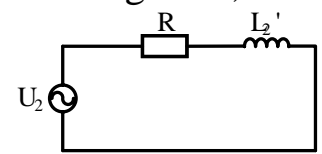

Fig. 3 Schematic diagram of cable and sea water equivalent circuit

As shown in figure $\mathrm{R}$ is the total circuit resistance, $\mathrm{L}_{2}$ ' is the inductance of the cable through the magnetic ring 2. Assuming that the output load of $U_{o}$ is infinite, by

$$
U_{i} \approx E_{1}=-N_{1} \frac{d \phi_{1}}{d t}, \quad U_{2} \approx E_{2}=-\frac{d \phi_{1}}{d t}
$$

You can get $\mathrm{U}_{\mathrm{i}}=\mathrm{N}_{1} * \mathrm{U}_{2}$, and $I_{2}=\frac{U_{2}}{Z_{2}}$

$$
Z_{2}=R+j \omega L_{2}^{\prime}=\left(R^{2}+(\omega \mathrm{L})^{2}\right)^{1 / 2} e^{j \arctan \frac{R}{\omega L}}
$$

According to the Ampere circuital theorem: $I_{2}=\oint \boldsymbol{H} \cdot d \boldsymbol{l}$,

$$
\phi_{2}=\int_{0}^{h} \int_{r_{1}}^{r_{2}} B \cdot d r d z \text { and } B=\mu H
$$

Hence

$$
\begin{aligned}
U_{o} & =-N_{3} \frac{d \phi_{2}}{d t}=-\frac{N_{3}}{N_{1}} \cdot \frac{\omega \mu h \ln \frac{r_{2}}{r_{1}}}{2 \pi \sqrt{R^{2}+\left(\omega \mathrm{L}_{2}^{\prime}\right)^{2}}} U_{m} \cos \left(\omega \mathrm{t}-90^{\circ}\right) \mathrm{e}^{-j \arctan \frac{R}{\omega \mathrm{L}_{2}^{\prime}}} \\
& =-\frac{N_{3}}{N_{1}} \cdot \frac{1}{\sqrt{\frac{R^{2}}{\left(\omega \mathrm{L}_{2}^{\prime}\right)^{2}}+1}} U_{m} \cos \left(\omega \mathrm{t}-90^{\circ}\right) \mathrm{e}^{-j \arctan \frac{R}{\omega \mathrm{L}_{2}^{\prime}}}
\end{aligned}
$$

It can be seen that the output amplitude $\mathrm{N}_{3} / \mathrm{N}_{1}$ is proportional to the number of turns of the input, and it decreases with the increase of $1 / \omega$, moreover, the absolute phase of the signal is changed.

\section{Communication System Design}

System used FPGA to achieve DPSK modulation, coding, synchronization and demodulation, it's easy to change and debug. The overall block diagram of the inductive coupling information transmission system is shown in Figure 4.

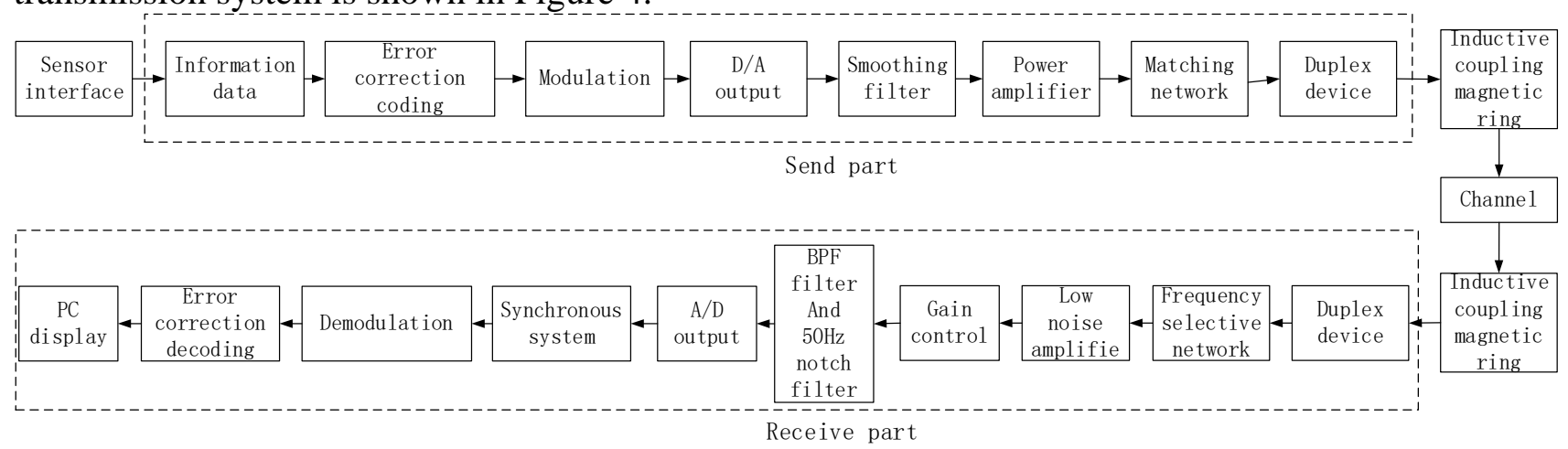

Fig. 4 Overall block diagram of information transmission in communication system

The hardware of transmitting and receiving system is mainly composed of several parts: power supply, induction coil, FPGA and its configuration circuit, AD/DA analog digital conversion module, 
power amplifier, receiver front-end, and data interface circuit.

Due to the presence of interference and attenuation, the data of the underwater instrument (sensor) cannot be transmitted by the baseband signal. As a result, there must be a process of modulation and demodulation for sending and receiving data [5]. It can be seen from the formula derivation, the carrier signal carrying the data information is sent out to the water surface through two coupling. The amplitude of the carrier signal is changed by the coupling, as well as the absolute phase shift. However, the frequency of $U_{0}$ and $U_{i}$ dose not change, the relative phase shift of the data symbols will not change too. This shows that the modulation of the carrier could use FSK or PSK, it's reasonable and reliable. In the case of the same symbol rate, the frequency band occupied by 2DPSK is narrower than that of 2FSK. That is, phase-discrete 2FSK band utilization is low, and the system efficiency is low. In the coherent demodulation and non-coherent demodulation, the coherent demodulation method is superior to the non-coherent demodulation method. Under the same bit error rate condition, the 2PSK has the minimum requirement of SNR. Figure 5 is a common binary digital modulation system BER curve.

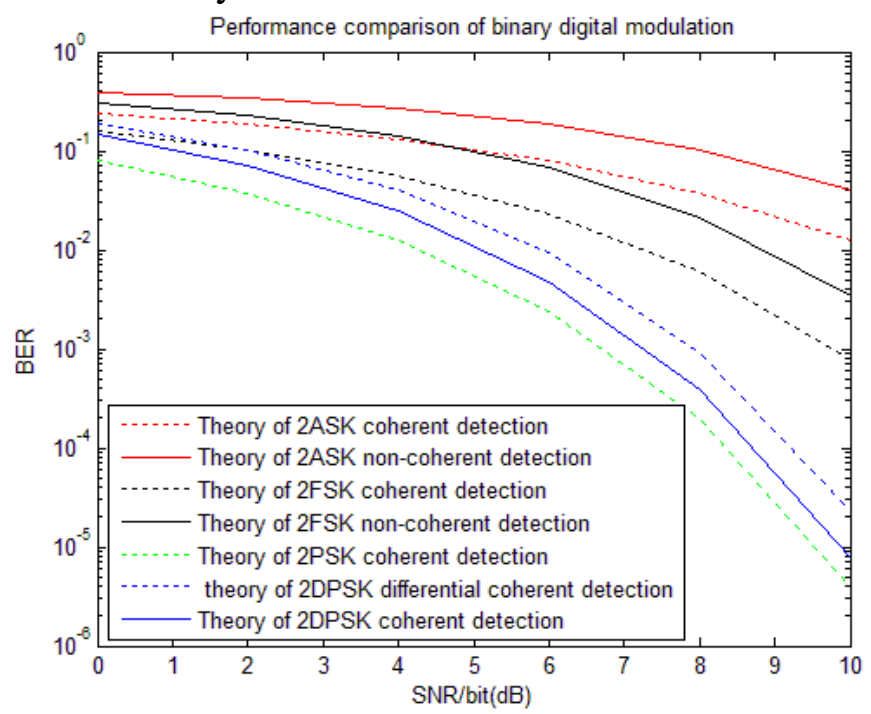

Fig. 5 Pe r curve of three kinds of digital modulation

Therefore, in order to obtain a good communication system performance, considering the complexity of the system, the 2DPSK modulation and demodulation method is adopted. According to the characteristics of the environment, the information rate and the carrier modulation frequency are determined to meet the requirements of the data transmission rate, such as the data of the sensor and image.

Channel coding can improve the reliability of communication, and the cost is the increase of the information redundancy and equipment complexity. In this paper, the system uses RS code. In the linear block code, the error correcting capability and coding efficiency of RS codes are the highest. Compared to other linear block codes in the same efficiency, error correcting capability of RS is particularly strong, its performance is close to the theoretical limit in the short and medium code length. What's more, there are strict algebraic structures, it's easy to construct, encoding and decoding is also simple.

\section{Experimental Test}

Firstly, the basic principle was tested. As shown in figure 6, this is a wire through two magnetic rings which connected end-to-end via a rheostat. A sinusoidal signal of $2 \mathrm{Vp}-\mathrm{p}$ was applied to one of the magnetic rings by a signal source, when the rheostat resistance was zero, it could be seen a peak value of the received signal on the other side of $1.38 \mathrm{Vp}-\mathrm{p}$, and it turned to be $45.6 \mathrm{mVp}-\mathrm{p}$ while the rheostat resistance increased to $200 \Omega$. It can be seen that with the increase of the loop resistance, the amplitude of received signal decreases. However, the loop resistance is limited, received signal will not too small to detect. So the way of inductive coupling communication is feasible, it could be 
realized after some processing.

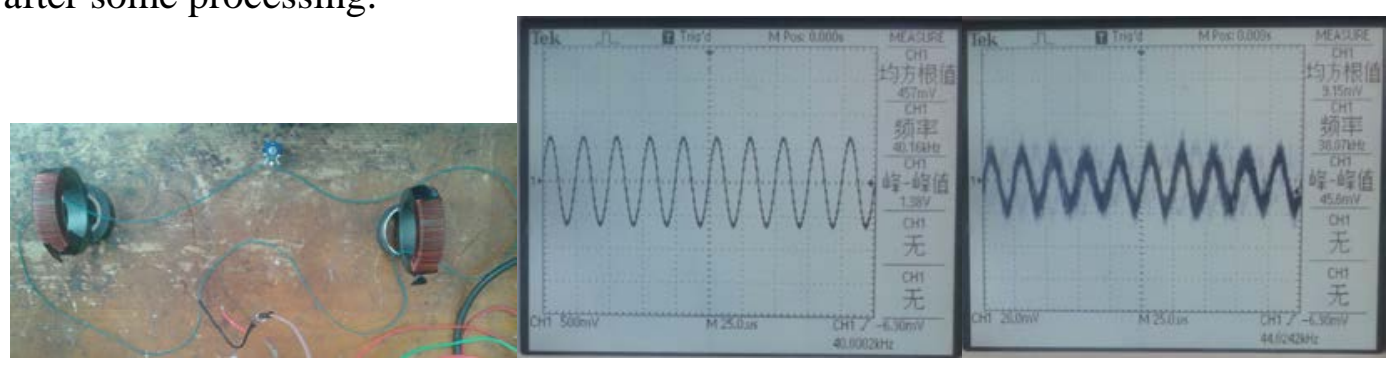

Fig. 6 Experimental results

Figure 8 shows a communication system used to test the effective transmission of data.

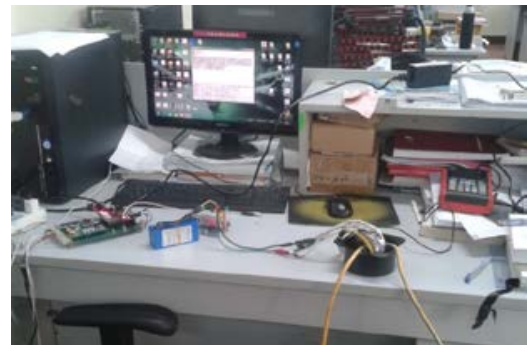

Fig. 7 Data transmitter

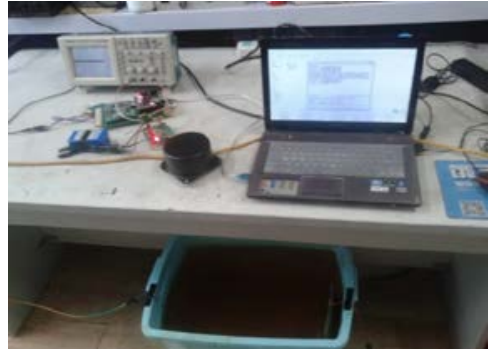

Fig. 8 Water tank and receiver.

\section{Conclusions}

In this paper, based on the analysis of the principle of inductive coupling communication, the system design experiments were carried out to achieve the basic communication goals. System used FPGA to achieve modulation and demodulation, channel coding, etc., it was conducive to improving system reliability. The system can be used to realize the real-time transmission of underwater sensor data, and it has a certain value of research.

\section{Acknowledgements}

This research has been supported by International Science and Technology Cooperation Program of China (2014 DFR10240), Hei Long Jiang Postdoctoral Foundation (LBH-Z14066), Science Foundation of Heilongjiang Province QC2015075, and Fundamental Research Funds for the Central Universities GK2080260146.

\section{References}

[1] Real-Time Oceanography with Inductive Moorings and the Inductive Modem Module (IMM) Sea-Bird Electronics, Inc. Revised May 2016.

[2] N. L. Brown, "Underwater sensors with inductive coupling to the sea cable," Proceedings, National Telemetry Conference, p. 185-191, 1965.

[3] Fougere, A.J, Brown, N.L, Hobart, E. Inductive Modem for Ocean Data Telemetry[C]// Oceans. 1991:1165-1170.

[4] SHAO Yi, LI Jianguo, LI Jiashun, et al. Temperature and salinity chain system for light-induced coupling data transmission [J]. OCEAN TECHNOLOGY, 2009, 28 (3): 36-39.

[5] Sklar B. Digital Communication: Fundamentals and Applications [M]. Publishing House of Electronics Industry, 2006. 